\title{
EDITORIAL
}

\section{Exposure assessment using biomonitoring: Is its usefulness fully realized?}

Journal of Exposure Science and Environmental Epidemiology (2007) 17, 307; doi:10.1038/sj.jes.7500582

Exposure assessment is a critical component of exposure science. Among the techniques that can be used for exposure assessment, biomonitoring is increasingly becoming one of the most popular choices. Biomonitoring, defined for the purposes of this special issue, is simply the measurement of a chemical, its metabolite, or reaction product in biological matrices, most commonly urine and blood products. Until the turn of the century, general population-based biomonitoring data were scarce. However, with the advent of largescale longitudinal epidemiologic studies, such as those conducted at the NIEHS/EPA's Children's Centers for Environmental Health and Disease Prevention and crosssectional general population biomonitoring studies published in the Centers for Disease Control and Prevention's National Reports on Human Exposure to Environmental Chemicals, volumes of data evaluating "background" or real-world exposures in the general population or selected subpopulations have become increasingly available. In addition, improvements in analytical equipment and methodology have allowed us to detect more chemicals at levels approaching the low parts per quadrillion (femptogram/ gram) level, in some instances. Our understanding of these levels and what they mean in terms of exposures and potential health risks have lagged far behind our ability to create massive amounts of biomonitoring data. Several organizations have spent a lot of time gathering expert information on how to best interpret biomonitoring data and utilize it to its fullest extent. This has been a major goal of the American Chemistry Council's (ACC) Long Range
Initiative and the theme of a workshop they held last year which included representatives from academia, government, and industry.

We are fortunate to have obtained funding from the ACC to produce this special issue on biomonitoring with Tina Bahadori as a guest editor. Dr. Bahadori, along with several of her colleagues, developed a report summarizing the main points derived from last year's ACC meeting. In addition, nine other papers are presented in this special issue that deal with some aspect of biomonitoring - from presenting general population measurements or interpretations, to investigating predictors of biomonitoring levels and including papers that deal with problems that have plagued the interpretation of biomonitoring data. Together, this special issue of the Journal of Exposure Science and Environmental Epidemiology presents an interesting set of papers detailing the complexity of deriving and interpreting biomonitoring measurements and attempts to utilize the full potential of these measurements. This issue represents the first of a series of steps that will be taken by exposure scientists to put biomonitoring measurements into context, and perhaps, use them to implement or evaluate regulatory actions, potential health risks, and ways of mitigating exposures. We gratefully acknowledge the funding that ACC has provided to produce this special collection for our readership.

DANA B. BARR Editor-in-Chief JESEE 